\title{
Study on Treatment of Harbour Oily Wastewater by Inorganic Ceramic Membrane
}

\author{
Yanjie Wei
}

Key Laboratory of Environmental Protection in Water Transport Engineering Ministry of Communications, Tianjin Research Institute of Water Transport Engineering, Tianjin 300456, China

\begin{abstract}
The inorganic ceramic membrane technology was utilized to treat harbour oily wastewater. The operational parameters were chosen and optimized. Finally, with the200nm- membrane module, the optimum parameters were obtained: operation pressure was $0.10 \mathrm{MPa}$, operational temperature was $303 \mathrm{~K}$, surrounding $\mathrm{pH}$ was around 6.77 , and crossflow velocity was $10 \mathrm{~m} / \mathrm{s}$. Under these operational parameters, the oil content could be removal remarkably under the influent content of $4 \sim 26 \mathrm{mg} / \mathrm{L}$, with the removal efficiency more than $98 \%$.
\end{abstract}

\section{Introduction}

Inorganic ceramic membrane has the advantages of high temperature resistance, high acid and alkali resistance, homogenous pore-size distribution, steady chemical property, high strength, large flux, long lifetime, antipollution, simple structure, small floor area, few matching equipment, easy installation, no chemical additives, high operating efficiency, high management and automation, and so on. Thus, this technology has been applied in the wastewater treatment of food industry, biochemical engineering, petrochemical industry, mechanical metallurgy. In this study, the inorganic ceramic membrane technology was utilized to treat harbour oily wastewater. The operational parameters were chosen and optimized.

\section{Experimental set-up}

A laboratory-scale inorganic ceramic experimental device (Figure 1, length $\times$ width $\times$ height $=1100 \times 600 \times 1300 \mathrm{~mm}$, Jiangsu Jiuwu Hi-Tech Co., Ltd., Nanjing China) was used in this study. A $\mathrm{ZrO}_{2}$ ceramic membrane module was submerged.The area of single membrane is $0.1 \mathrm{~m}^{2}$.

The system was fed with real harbor oily wastewater coming from a local harbor oily wastewater treatment plant in Tianjin Harbor, China. Influent of MBR had been pre-treated by coagulation-sedimentation process. Composition of the influent fed to the system is summarized in Table 1. COD, oil content, Cl- content, SS and MLSS were measured regularly according to the Standard Methods [1]

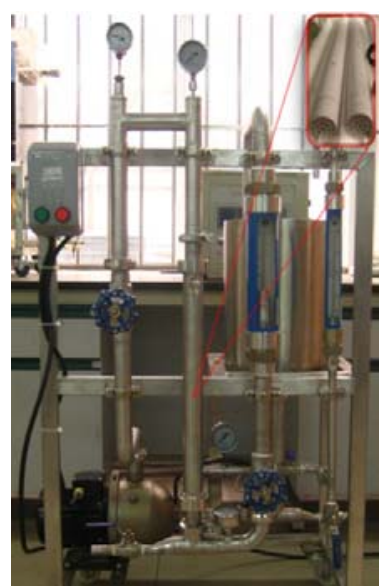

Figure 1. A laboratory-scale inorganic ceramic experimental device.

Table 1. Characteristics of influent wastewater

\begin{tabular}{|c|c|}
\hline Item & Feed \\
\hline $\mathrm{COD}\left(\mathrm{mgO}_{2} / \mathrm{L}\right)$ & $732.7 \pm 262.9$ \\
\hline oil content $(\mathrm{mg} / \mathrm{L})$ & $48.2 \pm 13.1$ \\
\hline $\mathrm{pH}$ & $7.13 \sim 8.08$ \\
\hline $\mathrm{SS}(\mathrm{mg} / \mathrm{L})$ & $55.3 \sim 352.1$ \\
\hline $\mathrm{Cl}^{-}(\mathrm{mg} / \mathrm{L})$ & $3300 \sim 3680$ \\
\hline
\end{tabular}

\section{Results and discussion}

\subsection{Membrane pore size}

Separation mechanism of the inorganic ceramic membrane mainly consist of screening. Thus, the proper membrane pore size should be chosen firstly.

The 50nm- and 200nm- membrane module were adopted to treat the real harbour oily wastewater. The performaces were deplcted in Fig. 2. It could be seen that the flux of 50nm-membrane module was only half of the one of 200nm-membrane module, whose initial flux was $410 \mathrm{~L} /\left(\mathrm{m}^{2} \cdot \mathrm{h}\right)$ and decresed to $184 \mathrm{~L} /\left(\mathrm{m}^{2} \cdot \mathrm{h}\right)$ in 20 mintiues, and finally stablized at $173 \mathrm{~L} /\left(\mathrm{m}^{2} \cdot \mathrm{h}\right)$. While, the initial and stable flux of $50 \mathrm{~nm}$-membrane module was lower than the one of $200 \mathrm{~nm}$-membrane module throughout the experitmental period. Based on these results, the 200nmmembrane module was chosen and used for the watewwater treatment in this study. 


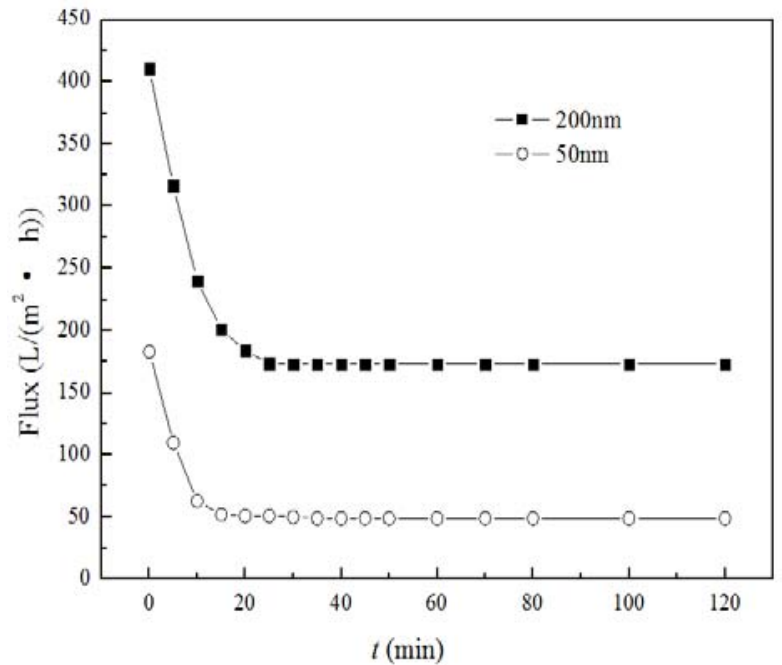

Figure 2. Effect of pore size on membrane flux ( $\mathrm{T}=298 \mathrm{~K}$, $u=10 \mathrm{~m} / \mathrm{s}, \Delta P=0.10 \mathrm{MPa}$ ）.

\subsection{Optimizing parameters}

\subsubsection{Operation pressure}

Operation pressure is the driving force for membrane filtration, and plays the key role on membrane flux. The relationship between operation pressure and membrane flux was studied and shown in Fig. 3.

A linear increase in membrane flux was noted with increasing operation pressure, when the pressure was $0.030 \mathrm{Mpa} 0.100 \mathrm{Mpa}$. This phase is typical pressure regime. When the pressure was between $0.100 \mathrm{MPa}$ to $0.165 \mathrm{Mpa}$, the increasing rate membrane flux declined due to the effect of concentration polarization.

Some researchers have identified that dense gel layer formed when the pressure increased continuously. The driving force became density control rather than pressure control. The critical point was about $0.1 \sim 0.2 \mathrm{MPa}$ [2]. It was also reported that a lot of pollutants entered into the membrane pores under the pressure over $0.25 \mathrm{Mpa}$, leading to difficulty of cleaning and regeneration.

Therefore, the optimum operation pressure was selected at $0.10 \mathrm{MPa}$

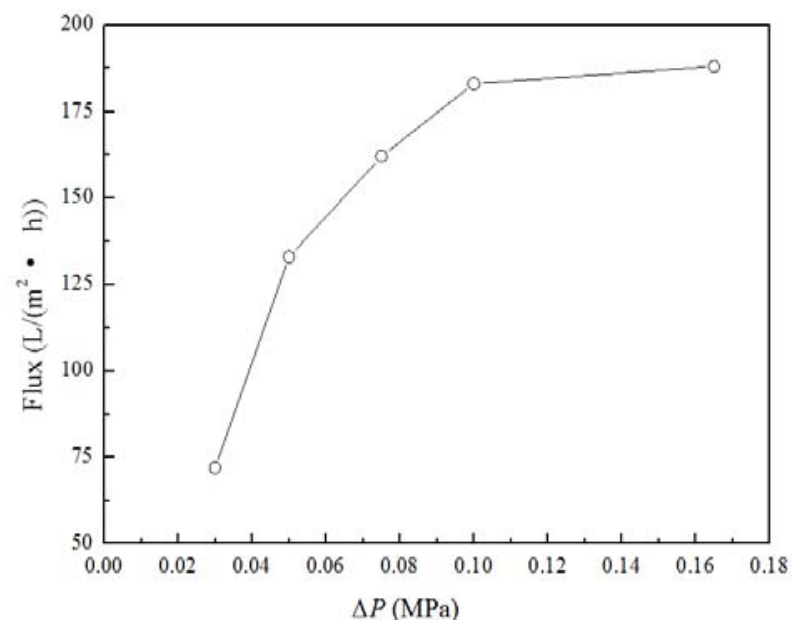

Figure 3. Effect of operation pressure on membrane flux （T=298K, $u=10 \mathrm{~m} / \mathrm{s}, \Delta P=0.10 \mathrm{MPa}$ ）.

\subsubsection{Temperature}

The flux can be significantly improved if the temperature increases, mainly due the following reasons [3]:

(1) Solution viscosity decreases with the temperature increasing. Remained shell on the membrane becomes thin, the flow state could be improved.

(2) Solute diffusion coefficient increases under the higher temperature. Solute adhered to the membrane surface can easily return to the bulk, contributing to alleviation of concentration polarization.

(3) The resistance passing the membrane pores can be cut down when the temperature increases.

Fig. 4 shows the effect of temperature on membrane flux. A proportional relationship between the temperature and flux was observed. However the higher operational temperature would also result in higher energy consumption and higher operation costs. Therefore, the proper operational temperature of 303K was adopted.

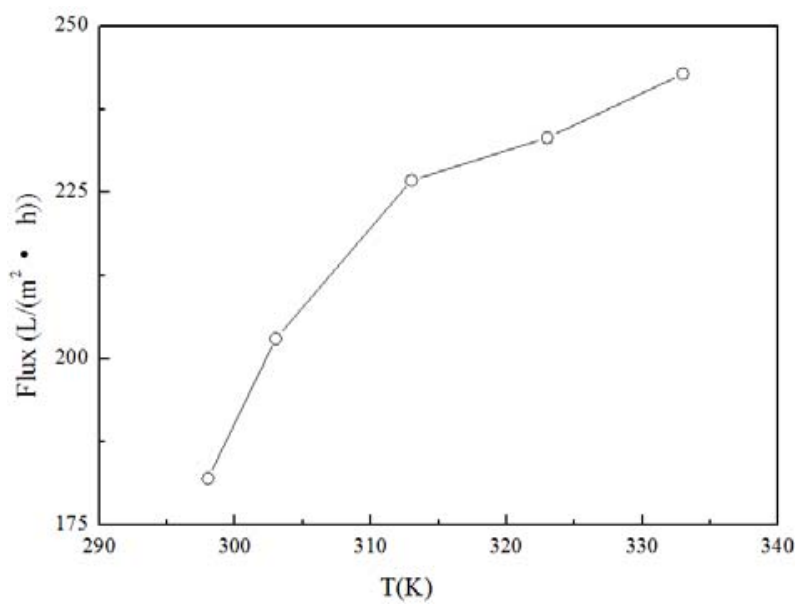

Figure 4. Effect of ambient temperature on membrane flux ( $\Delta$ $P=0.10 \mathrm{MPa}, u=10 \mathrm{~m} / \mathrm{s}$ ).

\subsubsection{Surrounding $\mathrm{pH}$}

$\mathrm{pH}$ is thought to be the most important environmental parameters for filtration. Because the surrounding $\mathrm{pH}$ is likely to determine the electrical properties of oil pellets and $\mathrm{ZrO}_{2}$ membrane, and affect the microfiltration process.

The relationship between the surrounding $\mathrm{pH}$ and membrane flux was studied, and the results were shown in Fig.5. It was found that the highest flux was achieved under the operational $\mathrm{pH}$ of 6.77. When the $\mathrm{pH}$ further increased, the flux began to decline. This phenomenon may be explained by the following: The isoelectric point of $\mathrm{ZrO} 2$ is 5 . When the surrounding $\mathrm{pH}$ is around 5, the flux reaches the maximum value. 


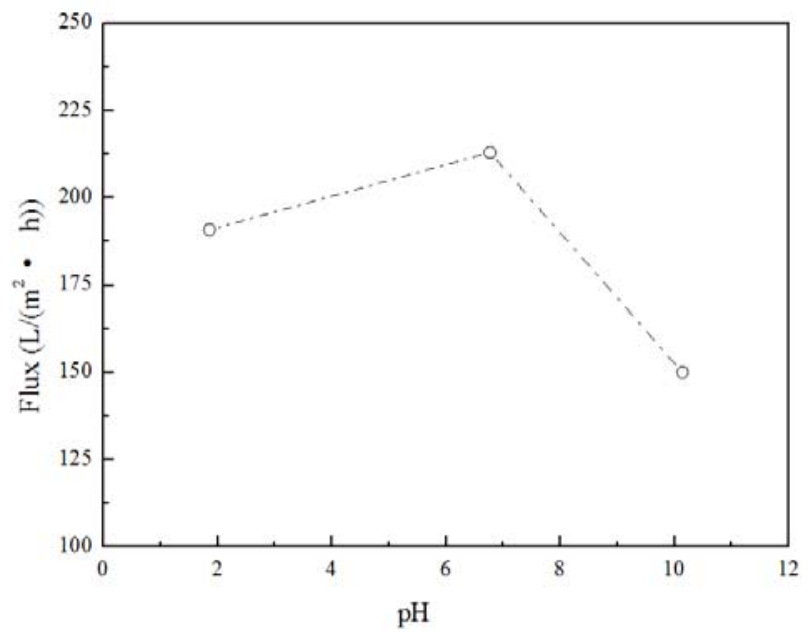

Figure 5. Effect of surrounding $\mathrm{pH}$ on membrane flux （T=303K, $u=10 \mathrm{~m} / \mathrm{s}, \Delta P=0.10 \mathrm{MPa}$ ）.

\subsubsection{Crossflow velocity}

It is known that improvement of hydrodynamic condition in membrane systems, including crossflow velocity along membrane surface, can effectively mitigate membrane fouling [4]. Fig.6 shows the effect of crossflow velocity on membrane flux. It could be seen that the flux increased with the increment of crossflow velocity, when the velocity was between $6.3 \mathrm{~m} / \mathrm{s}$ to $10.0 \mathrm{~m} / \mathrm{s}$. This phenomenon could be explained that oil pellets can be effectively removed by the shear stress after improving the crossflow velocity. The thickness of the gel layer on the membrane surface decreased, contributing to alleviation of concentration polarization and improvement of flux.

However, when the crossflow velocity was over $10.0 \mathrm{~m} / \mathrm{s}$, the increasing rate of flux declined. The observation could probably be explained by the fact that flowing resistance increases and the retention time of solution adhering to the membrane surface is shortened, after further increment of crossflow velocity. These conditions do not benefit for mass-transfer.

Thus, the crossflow velocity of $10 \mathrm{~m} / \mathrm{s}$ was adopted.

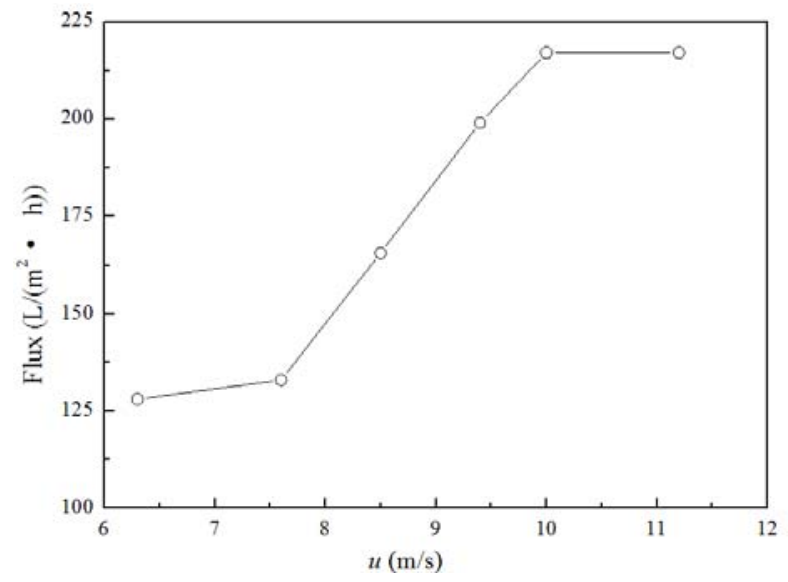

Figure 6. Effect of crossflow velocity on membrane flux $(\mathrm{T}=303 \mathrm{~K}, \Delta \mathrm{P}=0.10 \mathrm{MPa})$

\subsection{Removal performances}

The laboratory-scale inorganic ceramic experimental device was used to treat real harbour oily wastewater with the optimum parameters selected above. Pollutants removal performance was shown in Fig. 7. It could be found that the removal efficiencies were poor. When the influent COD was low $(200 \sim 530 \mathrm{mg} / \mathrm{L})$, the removal efficiency was only about $40 \%$.

The device presented excellent performance on reducing the oil content. When the influent concentration was $4 \sim 26 \mathrm{mg} / \mathrm{L}$, the effluent value was lower than 0.45 $\mathrm{mg} / \mathrm{L}$, with the removal efficiency over $98 \%$.
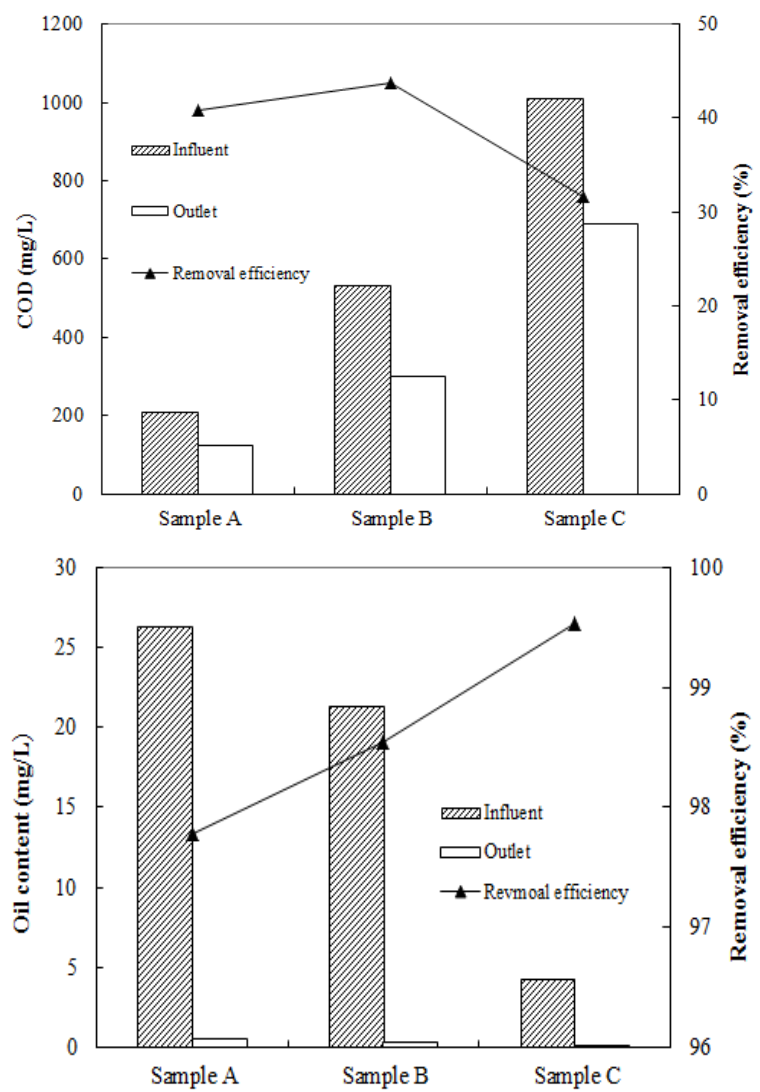

Figure 7. Pollutants removal performances

\section{Conclusion}

Ceramic membrane technology has become one of the most important technologies treating oily wastewater.

The inorganic ceramic membrane with the 200nmmembrane module was utilized to treat real harbour oily wastewater. The operational parameters were optimized, with the operation pressure of $0.10 \mathrm{MPa}$, operational temperature 303K, surrounding $\mathrm{pH} 6.77$, and crossflow velocity $10 \mathrm{~m} / \mathrm{s}$. Under these operational parameters, the oil content could be removal remarkably. When the influent concentration was $4 \sim 26 \mathrm{mg} / \mathrm{L}$, the effluent value was lower than $0.45 \mathrm{mg} / \mathrm{L}$, with the removal efficiency over $98 \%$. 


\section{References}

[1] APHA,. Standard Methods for the Examination of Water and Wastewater (20th Edition. American Public Health Association, Washington, DC, USA, 1998)

[2] S.L. Li, The establishment of model and simulation study of microfiltration for dispersions using Tu bular membrane (Dalian University of Technology, 2006)

[3] J.X., Zeng, L.F., Zhang, H.Q., Ye, et al., The Chinese Journal of Process Engineering 10, 3 (2010)

[4] P., Bacchin, B., Espinasse, 2005. Journal of Membrane Science, 250, 1-2(2005) 